\title{
Subcellular Mechanisms of Presenilin-Mediated Enhancement of Calcium Signaling
}

\author{
Malcolm A. Leissring,* Frank M. LaFerla, ${ }^{* 11}$ Nick Callamaras, ${ }^{+}$ \\ and lan Parker ${ }^{\dagger}$ \\ * Laboratory of Molecular Neuropathogenesis and ${ }^{\dagger}$ Laboratory of Cellular and Molecular \\ Neurobiology, Department of Neurobiology and Behavior, University of California at Irvine, \\ 1109 Gillespie Neuroscience Research Facility, Irvine, California 92697-4545
}

Received September 26, 2000; revised November 17, 2000; accepted J anuary 4, 2001;

published online May 3, 2001

\begin{abstract}
Mutations in presenilin-1 (PS1), the leading cause of early-onset, autosomal-dominant familial Alzheimer's disease (FAD), enhance calcium signaling mediated by inositol 1,4,5-trisphosphate (IP ${ }_{3}$ ). To elucidate the subcellular mechanisms underlying this enhancement, we used high resolution linescanning confocal microscopy to image elementary calcium release events ("puffs") in Xenopus oocytes expressing wild-type or mutant PS1. Here we report that mutant PS1-rendered puffs more sensitive to $I P_{3}$ and increased both the magnitude and the rate of calcium release during each event. These effects were not attributable to quantitative changes in the levels of $\mathrm{IP}_{3}$ receptors or their distribution on the ER, but were instead associated with an abnormal elevation of ER calcium stores. Together, our results suggest that the effects of mutant PS1 on calcium signaling are manifested predominantly at the level of the regulation of calcium stores rather than via perturbations in the numbers or activity of $\mathrm{IP}_{3}$-activated calcium release channels. $\odot 2001$ Academic Press

Key Words: Alzheimer's disease; calcium; confocal microscopy; endoplasmic reticulum; phosphoinositide; presenilin; Xenopus oocytes.
\end{abstract}

\section{INTRODUCTION}

Presenilin 1 and 2 (PS1, PS2) are highly conserved, pleiotropic proteins localized predominantly to the endoplasmic reticulum (Kovacs et al., 1996; Weber et al., 1997; Zhang et al., 1998). Mutations in the presenilins cause a number of cellular defects implicated in the pathogenesis of Alzheimer's disease, including altered $\gamma$-secretase-mediated cleavage of the $\beta$-amyloid precursor protein (APP) and dysregulation of intracellular calcium signaling pathways (for reviews see Mattson et al., 2000a; Selkoe, 1999). Although the mechanisms by which presenilin mutations lead to APP mismetabolism have been extensively studied, relatively little is known about the precise subcellular disturbances underlying the effects of presenilin mutations on calcium dyshomeostasis.

\footnotetext{
${ }^{1}$ To whom correspondence and reprint requests should be addressed. Fax: (949) 824-7356. E-mail: laferla@uci.edu.
}

Like effects on APP processing, calcium signaling alterations appear to be an early and invariant consequence of presenilin mutations, having been documented for multiple mutations in PS1 and PS2 in numerous experimental systems (Barrow et al., 2000; Etcheberrigaray et al., 1998; Gibson et al., 1996; Hirashima et al., 1996; Ito et al., 1994; Keller et al., 1998; Leissring et al., 2000a; Leissring et al., 1999a; Leissring et al., 1999b, 2000b; Mattson et al., 2000b; Parent et al., 1999). For instance, even prior to the identification of the presenilins, calcium alterations were described in skin fibroblasts from a family of chromosome 14linked FAD patients, who were later shown to harbor the A246Q mutation in PS1 (Ito et al., 1994). Intriguingly, these perturbations in calcium signaling are so selective and so consistent that they can be used to reliably predict FAD several years prior to presentation of overt neurological symptoms (Etcheberrigaray et al., 1998). Recently, we demonstrated that nearly identical disturbances are present in fibroblasts from mutant $P S 1_{M 146 V}$ knock-in animals (Leissring et al., 2000a), 
providing further evidence that perturbed calcium signaling is an early and likely pathogenic event conferred by presenilin mutations. Finally, we have shown that mutations in both PS1 and PS2 cause indistinguishable changes in calcium signaling (Leissring et al., 1999a, 1999b), suggesting that these alterations that are a common pathogenic consequence of all presenilin mutations.

Studies on the effects of presenilin mutations on calcium signaling have predominantly focused on the phosphoinositide signal transduction pathway, in which calcium is liberated from the ER into the cytosol through receptor/channel complexes activated by the second messenger inositol 1,4,5-trisphosphate (IP Etcheberrigaray et al., 1998; Gibson et al., 1996; Ito et al., 1994; Leissring et al., 2000a). The specific constellation of disturbances conferred by presenilin mutations include (i) a potentiation of calcium release from the ER (Etcheberrigaray et al., 1998; Gibson et al., 1996; Ito et al., 1994; Leissring et al., 2000a, 1999a, 1999b, 2000b) and (ii) deficits in capacitative calcium entry (CCE; Leissring et al., 2000a; Yoo et al., 2000), an important signaling pathway wherein depletion of ER calcium stores triggers a sustained influx of extracellular calcium into the cytosol (Berridge, 1995). Although these alterations are now well described, and have proven diagnostic utility (Etcheberrigaray et al., 1998), the mechanisms underlying these changes are poorly understood.

Advances in imaging technology have begun to reveal the detailed mechanisms of ER calcium signaling. The advent of high-resolution forms of line-scanning confocal microscopy has revealed that global calcium signals, which are often graded in nature, are in fact comprised from elementary calcium release events of a fairly consistent size. These events, known as "puffs," arise from the concerted opening of clusters of $\mathrm{IP}_{3}$ receptors, and are thought to represent the fundamental building blocks of global calcium signals (Berridge, 1997; Parker et al., 1996; Sun et al., 1998). Because calcium puffs are discrete events, highly localized both in space and in time, the fine-scale kinetics of calcium release can be studied in exquisite detail (Sun et al., 1998). In the present study, we used high resolution confocal microscopy to elucidate the mechanisms by which presenilin mutations alter phosphoinositide calcium signaling using the Xenopus oocyte, a system that has been exploited extensively to characterize proteins relevant to neuronal function and the detailed mechanisms of ER calcium signaling. We report that oocytes overexpressing mutant PS1 generate puffs at lower $\left[\mathrm{IP}_{3}\right]$ than control cells or cells overex- pressing wild-type (wt) PS1 and that the rate and total amount of calcium liberation from the ER during these elementary events are enhanced. We provide evidence that these changes are attributable to abnormal elevation of calcium within the ER.

\section{METHODS}

\section{CRNA Synthesis and Injection}

Full-length cDNAs encoding human wtPS1 and $\mathrm{PS}_{\mathrm{M} 146 \mathrm{~V}}$ were the generous gift of Dr. John Hardy (Clark et al., 1995). Synthesis of $\mathrm{m}^{7} \mathrm{G}\left(5^{\prime}\right) \mathrm{ppp}\left(5^{\prime}\right) \mathrm{G}-$ capped cRNA was performed by run-off transcription of template plasmids linearized with the restriction endonuclease XbaI using the Riboprobe Gemini System (Promega) according to the manufacturer's recommendations. The quantity and quality of the resulting transcripts were determined using spectrophotometric analysis and direct visualization on a denaturing gel (Leissring et al., 1999b). Stage V and VI oocytes from Xenopus laevis (Xenopus I, Ann Arbor, MI) were defolliculated by two 1-h treatments with 0.5 $\mathrm{mg} / \mathrm{ml}$ Type I collagenase (Sigma) and were injected the following day with $46 \mathrm{nl}$ of the appropriate cRNA (500 ng/ $\mu \mathrm{l}$ ) or RNase-free $\mathrm{H}_{2} \mathrm{O}$. Oocytes were maintained in Barth's solution, containing (in $\mathrm{mM}$ ) $\mathrm{NaCl}$ (88), $\mathrm{KCl}(1), \mathrm{NaHCO}_{3}(2.4), \mathrm{MgSO}_{4}(0.83), \mathrm{Ca}\left(\mathrm{NO}_{3}\right)_{2}$ (0.33), $\mathrm{CaCl}_{2}$ (0.41), Hepes (10), pH 7.4, supplemented with sodium pyruvate (550 mg/L) and gentamycin (50 $\mathrm{mg} / \mathrm{L})$.

\section{Loading with Calcium Indicator and $\mathrm{Caged}_{\mathrm{IP}} \mathbf{P}_{\mathbf{3}}$}

Three days after cRNA injection and $1-4 \mathrm{~h}$ prior to calcium imaging experiments, oocytes were loaded with $23 \mathrm{nl}$ of a mixture containing $0.5 \mathrm{mM}$ caged $\mathrm{IP}_{3}$ (d-myo-inositol 1,4,5-trisphosphate, $\mathrm{P}^{4(5)}$-(1-(2-nitrophenyl)ethyl) ester), and $2 \mathrm{mM}$ of either the highaffinity calcium indicator Oregon Green BAPTA-1 (OG-1) or the low-affinity indicator Oregon Green-5N (OG-5N). To ensure consistent loading of oocytes, we used a piston-driven Nanoject microinjector (Drummond Scientific) fitted with glass electrodes with tip diameters of $\sim 15-20 \mu \mathrm{m}$. Oocytes of a uniform size $(\sim 1.3 \mathrm{~mm})$ were selected prior to loading. Based on this size, the cytosolic volume is estimated to be $\sim 1 \mu \mathrm{l}$ (corrected for yolk granules and other organelles), yielding final concentrations in the oocytes of $\sim 12 \mu \mathrm{M}$ caged $\mathrm{IP}_{3}$ and $\sim 46 \mu \mathrm{M}$ calcium indicator. Calcium indicators and caged $\mathrm{IP}_{3}$ were obtained from Molecular Probes (Eugene, OR). 


\section{Photolysis of $\mathbf{C a g e d} \mathrm{IP}_{3}$ and $\mathrm{C}$ alcium Imaging}

Photolysis of caged $\mathrm{IP}_{3}$ to liberate free intracellular $\mathrm{IP}_{3}$ was achieved using flashes of UV light (340-400 $\mathrm{nm}$ ) derived from a mercury arc lamp, illuminating a circular portion of the oocyte $\sim 100 \mu \mathrm{m}$ in diameter (Leissring et al., 1999b). The amount of photoreleased $\mathrm{IP}_{3}$ is a linear function of the concentration of caged $\mathrm{IP}_{3}$ within the oocyte, the UV light intensity, and the flash duration (Callamaras \& Parker, 1998). Oocytes were loaded with a fixed amount of caged $\mathrm{IP}_{3}$ and the light intensity remained constant, so that the final concentration of photoreleased $\mathrm{IP}_{3}$ could be regulated consistently in different oocytes by varying the flash durations with an electromechanical shutter. The initial intensity of the UV source was set by adjusting a neutral-density filter in the light path to a setting that yielded a reasonable working range of flash durations. For experiments designed to evoke calcium puffs, the concentrations of $\mathrm{IP}_{3}$ evoked by these flashes are estimated to be somewhat below $\sim 50 \mathrm{nM}$, the amount required to evoke calcium waves, in the range corresponding to subthreshold endogenous metabotropic receptor stimulation (Callamaras \& Parker, 1998). In experiments recording responses to supramaximal $\mathrm{IP}_{3}$ stimulation, the light intensity was increased 100-fold, and the aperture of the UV light source was increased to illuminate the maximum possible area of the oocyte. Calcium-dependent fluorescence changes were imaged using a custom-built line-scanning confocal microscope, and data were recorded and analyzed as described previously (Callamaras \& Parker, 1999). Unless otherwise noted, images were formed by scanning a $50 \mu \mathrm{m}$ line at a rate of $8 \mathrm{~ms}$ per line (or $20 \mathrm{~ms}$ per line in experiments involving supramaximal $\mathrm{IP}_{3}$ stimulation), with a nominal pixel size of $0.133 \mu \mathrm{m}$ and an integration time of $20 \mu$ s per pixel. All recordings were obtained with the scan line focused at the depth of the pigment granules in the oocyte (roughly $6 \mu \mathrm{m}$ below the surface), where $\mathrm{IP}_{3}$-sensitive release sites are concentrated (Yao et al., 1995). Fluorescence signals are expressed as ratios $\left(\Delta F / F_{\mathrm{o}}\right)$ of the change in fluorescence at each pixel during a response $(\Delta F)$ relative to the resting fluorescence at that pixel before stimulation $\left(F_{\mathrm{o}}\right)$. Mean values for $F_{\mathrm{o}}$ were obtained by averaging over several scan lines before the photolysis flash. OG-1 was used when imaging puffs, whereas maximal calcium responses were recorded using the lower-affinity indicator OG-5N. Most imaging experiments were performed in Ringer's solution, containing (in $\mathrm{mM}$ ) $\mathrm{NaCl}$ (120), $\mathrm{KCl}(2), \mathrm{CaCl}_{2}$ (1.8), Hepes (5), $\mathrm{pH}$ 7.4. Experiments estimating the relative size of ER calcium stores were conducted in calcium-free Ringer's solution, which contained $1 \mathrm{mM}$ EGTA and no $\mathrm{CaCl}_{2}$.

\section{Protein Immunoblotting}

Cells were frozen immediately after imaging for subsequent processing. Protein extracts were prepared as described (Leissring et al., 1999b) and concentrations determined by the Bradford method. Equal amounts of protein $(10 \mu \mathrm{g})$ were separated by SDSPAGE on $5 \%$ or $10 \%$ acrylamide gels, transferred to nitrocellulose membranes, blocked overnight in 5\% nonfat milk in Tris-buffered saline ( $\mathrm{pH}$ 7.5) supplemented with $0.2 \%$ Tween 20 , and processed as described previously (Leissring et al., 1999b). Presenilinspecific antibodies and dilutions used in this study included SW2 1:500; (Weber et al., 1997), amino- and carboxy-terminal fragment-specific antibodies (1:200; Chemicon, Temecula, CA), and PS1 $1_{\mathrm{NT}}$ and PS1 $1_{\text {cloop }}$ (1: 5000) (Thinakaran et al., 1996, 1998). Type-1 $\mathrm{IP}_{3}$ receptors were detected with rabbit polyclonal antibody $\alpha \mathrm{IP}_{3} \mathrm{R}-1$ (1:200; Affinity Bioreagents, Golden, CO). Quantitative densitometric analyses were performed on digitized images of immunoblots using the Stratagene EágleEye II gel documentation system according to the manufacturer's recommendations (Stratagene, La Jolla, CA).

\section{Data Analysis}

Calculation of the "signal mass" associated with calcium release events was accomplished using a custom routine written in the IDL programming language (Research Systems Inc., Boulder, CO) to integrate the one-dimensional linescan profile throughout three dimensions (Sun et al., 1998). Events were selected that appeared to involve calcium release from only a single site, were in sharp focus, and were well separated in time or space from other signals. The center of the event was identified visually and, for each time point (scan line), the routine then summed the fluorescence signals $\left(\Delta F / F_{\mathrm{o}}\right)$ along the scan line, with each value weighted by the third power of the distance of that pixel from the center. For convenience, the resulting sums were then scaled in units of $1 \Delta F / F_{\mathrm{o}} \times 10^{-15} 1$, representing a doubling in fluorescence throughout a volume of 1 femtoliter. Because of the third-power weighting, signal mass measurements showed greatly increasing noise and susceptibility to error from calcium originating at other sites as the integration was performed at increasing distances from the event cen- 


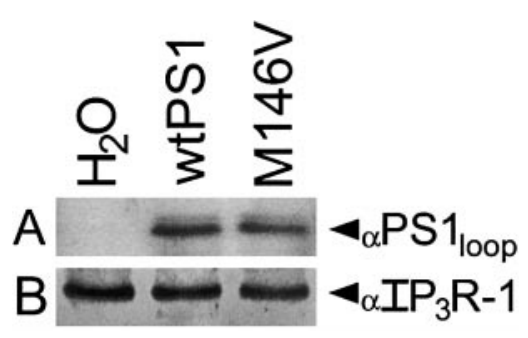

FIG. 1. Western analysis of expression of PS1 and $\mathrm{IP}_{3}$ receptors. Immunoblots show protein extracts from $\mathrm{H}_{2} \mathrm{O}$-injected control cells, and cells injected with equal amounts of wtPS1 or PS1 $1_{\mathrm{M} 146 \mathrm{~V}}$ cRNA. (A) Representative immunoblot of PS1 protein levels assayed using the antibody $\alpha \mathrm{PS} 1_{\text {loop }}$. Levels of wild type and mutant PS1 proteins differed by $<5 \%$, as determined by densitometric analysis of three independent Westerns (100 \pm 3.4 vs $98 \pm 4.3$, respectively, expressed in normalized arbitrary units \pm SE). Similar results were obtained using several other well-characterized anti-PS1 antibodies (see Methods). (B) Type-1 $\mathrm{IP}_{3}$ receptors, detected with antibody $\alpha \mathrm{IP}_{3} \mathrm{R}-1$ (Affinity Bioreagents), were expressed to comparable levels in the various conditions $(100 \pm 6.5,102 \pm 3.7$, and $99 \pm 5.0$ normalized arbitrary units, respectively, for control cells and cells expressing wtPS1 or PS1 $1_{\mathrm{M} 146 \mathrm{~V}}$ based on three independent Westerns).

ter. Consequently, calculations were restricted to a limited region of sufficient width to encompass virtually all of the signal, and the same width was used for measurements of signal mass from all experimental conditions.

Experiments were performed using 26-33 oocytes per condition, obtained from three different donor frogs. Statistical comparisons were made using twofactor ANOVA with replication. All results are presented as mean values \pm SE.

\section{RESULTS}

\section{Expression of Presenilin and $\mathrm{IP}_{3}$ Receptors}

Experiments were performed in Xenopus oocytes injected with equal amounts of cRNA encoding wild type PS1 (wtPS1) or the M146V mutation (PS1 $1_{\mathrm{M} 146 \mathrm{~V}}$ ), or with $\mathrm{H}_{2} \mathrm{O}$ as a control. Immunoblot analysis was used to monitor expression of the presenilin constructs in the oocytes used for imaging, and revealed that the wild-type and mutant PS1 proteins were present at similar steady-state levels (Fig. 1A). We also performed immunoblot analysis of type $1 \mathrm{IP}_{3}$ receptors, the only type of calcium release channel expressed in Xenopus oocytes; this cell type does not express ryanodine receptors (Kume et al., 1993). Our analysis revealed that $\mathrm{IP}_{3}$ receptor levels did not differ significantly among the experimental conditions (Fig. 1B), indicating that the potentiation of $\mathrm{IP}_{3}$-mediated calcium signals did not result through increased numbers of $\mathrm{IP}_{3}$ receptors/channels.

\section{Imaging of Calcium Puffs}

Figure 2 illustrates the basic imaging protocol, and shows representative records of $\mathrm{IP}_{3}$-mediated calcium signals in response to increasing $\left[\mathrm{IP}_{3}\right]$ produced by photolysis flashes of varying durations in a control cell (Figs. 2Aa-c) and a cell expressing $\mathrm{PS}_{\mathrm{M} 146 \mathrm{~V}}$ (Figs. $2 \mathrm{Ba}-\mathrm{c})$. In these linescan images, successive scans are stacked from left to right, so that distance along the scan line is depicted vertically, and time horizontally. Fluorescence is represented by a pseudocolor scale, with warmer colors indicating higher fluorescence intensities (higher free $\left[\mathrm{Ca}^{2+}\right]$ ). Brief photolysis flashes evoked discrete, localized calcium release events (puffs) at fixed points along the scan line, whereas longer flashes elicited calcium waves that are generated by the coordinated activation of many calcium release sites through successive cycles of calcium diffusion and calcium-induced calcium release (Berridge, 1997; Parker et al., 1996).

As illustrated in Fig. 2, oocytes expressing the $\mathrm{PS}_{\mathrm{M} 146 \mathrm{~V}}$ mutation exhibited several differences relative to control cells: puffs and waves were evoked at lower $\left[\mathrm{IP}_{3}\right]$; the puffs were of greater amplitude; and, as reported previously (Leissring et al., 1999b), responses to supramaximal $\left[\mathrm{IP}_{3}\right]$ were potentiated (Figs. $2 \mathrm{Ad}$ and 2Bd). These differences are described in greater detail in the following sections.

\section{Sensitization of $\mathrm{IP}_{\mathbf{3}}$-Evoked Calcium Release by Mutant PS1}

To quantify the sensitivity of oocytes to photoreleased $\mathrm{IP}_{3}$, we determined the threshold flash durations (i.e., relative concentrations of $\mathrm{IP}_{3}$ ) required to elicit puffs and waves. The minimum flash durations required to evoke at least one puff per linescan image in wtPS1 and PS1 ${ }_{\mathrm{M} 146 \mathrm{~V}}$-expressing cells $(n=15-17$ per condition) were, respectively, $84 \pm 12$ and $63 \pm 7 \%$ of that in controls (Fig. 3A). The flash durations required to evoke calcium waves were similarly reduced to $75 \pm 10$ and $56 \pm 7 \%$ of that of controls, respectively (Fig. 3B). Statistically, for both puffs and waves, $\mathrm{PS1}_{\mathrm{M} 146 \mathrm{~V}}$-expressing cells were significantly more sensitive to $\mathrm{IP}_{3}$ relative both to wtPS1-expressing cells and controls ( $P<0.05$ for both comparisons), while the latter two groups did not differ significantly from one another $(P>0.05)$. 

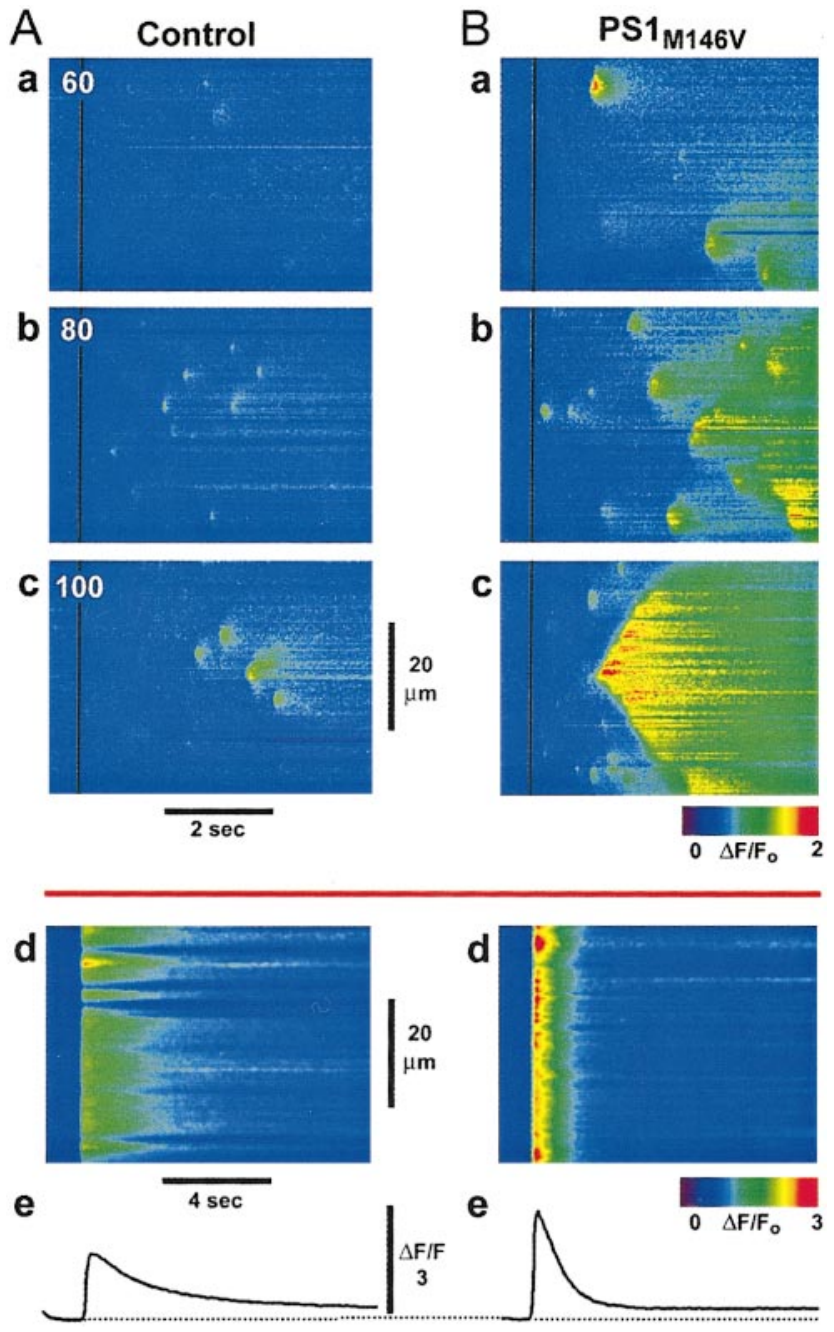

FIG. 2. Oocytes expressing mutant PS1 exhibit larger calcium signals and generate puffs and waves at lower $\left[\mathrm{IP}_{3}\right]$. Linescan images show representative records of calcium release evoked by increasing amounts of photoreleased $\mathrm{IP}_{3}$ in control cells (A) and cells expressing $\mathrm{PS}_{\mathrm{M} 146 \mathrm{~V}}(\mathrm{~B})$. Relative to control cells $(\mathrm{Aa}-\mathrm{C})$, mutant PS1-expressing cells $(\mathrm{Ba}-\mathrm{c})$ showed enhanced sensitivity and larger puffs in response to comparable photolysis flashes (durations indicated in $\mathrm{ms}$ ). Calcium waves evoked by supramaximal $\left[\mathrm{IP}_{3}\right]$ were also enhanced, as illustrated by the linescan images (Ad, Bd) and corresponding measurements of averaged fluorescence measured across the entire scan line $(\mathrm{Ae}, \mathrm{Be})$. Records were obtained using OG-1 as the indicator in $(\mathrm{a}-\mathrm{c})$ and $\mathrm{OG}-5 \mathrm{~N}$ in $(\mathrm{d}, \mathrm{e})$. Cells expressing wtPS1 exhibited responses intermediate between control and mutant PS1-expressing cells (data not shown).

\section{Potentiation of Calcium Puffs by PS1}

The amplitude of calcium puffs increases with increasing $\left[\mathrm{IP}_{3}\right]$ (Yao et al., 1995). To compare puffs between control and PS1-expressing cells, we therefore determined puff amplitudes (peak fluorescence
A

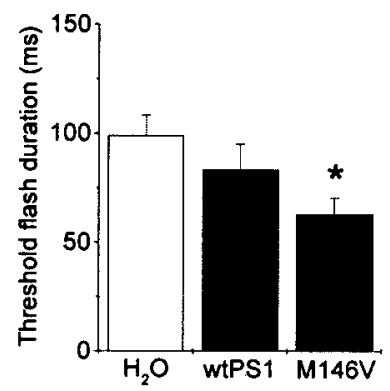

B

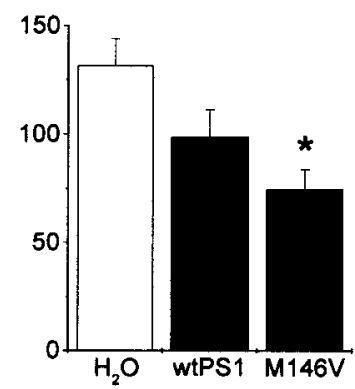

FIG. 3. Calcium release is sensitized by mutant PS1. Histograms plot the threshold flash durations (relative concentrations of $\mathrm{IP}_{3}$ ) required to elicit puffs (A) and waves (B) in control oocytes and oocytes expressing wild type and mutant PS1 $\left({ }^{*} P<0.05\right.$ relative both to controls and to wild type PS1-expressing cells; $n=15-17$ cells per condition.)

signals) over a range of flash durations for each condition (Fig. 4). Mean puff amplitudes in $\mathrm{PS}_{\mathrm{M} 146 \mathrm{~V}}-\mathrm{ex}-$ pressing cells were greater than in controls at corresponding flash durations, whereas wtPS1-expressing cells exhibited intermediate puff amplitudes. The relationship between puff amplitude and $\left[\mathrm{IP}_{3}\right]$ was markedly steeper in cells expressing $P S 1_{\mathrm{M} 146 \mathrm{~V}}$ relative to controls, while that of wtPS1-expressing cells more closely resembled control cells.

Measurements of fluorescence intensity correspond to the local free calcium concentration at the confocal spot, but do not indicate the amount of calcium liberated during release events. To estimate the latter, we

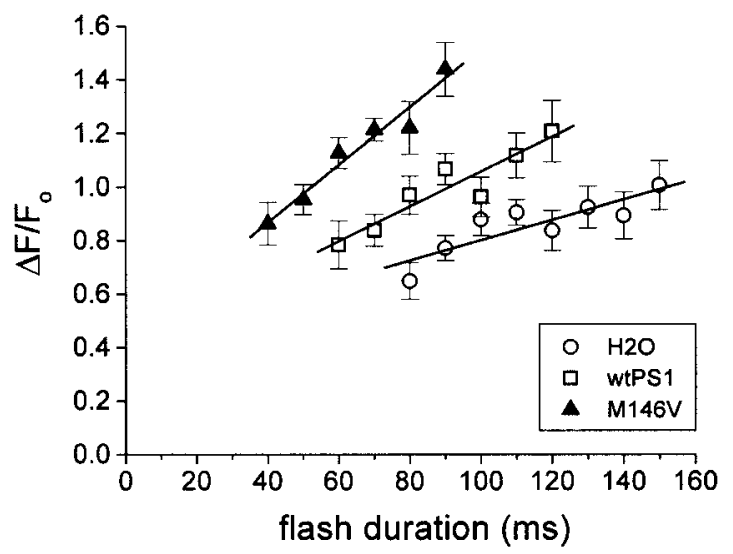

FIG. 4. Dependence of puff amplitude on $\left[\mathrm{IP}_{3}\right]$ in control and PS1-expressing oocytes. Graph shows mean peak fluorescence signals during puffs as a function of flash duration in control cells $(O)$, and cells expressing wild-type PS1 $(\square)$ and PS1 $1_{\mathrm{M} 146 \mathrm{~V}}(\boldsymbol{\Delta})$. Data were obtained from 15-17 cells per condition. 
calculated the "signal mass" associated with release events (see Methods). The calculation is based on the assumption that the laser scan line cuts through the center of a spherically symmetric cloud of calcium diffusing from a point source. Fluorescence along the scan line then represents a profile of calcium along a diameter of this sphere, and the total fluorescence at any time during an event can be obtained by integrating the profile through three dimensions. It is convenient to express the resulting signal mass in units of $\Delta F / F_{\mathrm{o}} \times 10^{-15} 1$; that is, one signal mass unit corresponds to a doubling of fluorescence within 1 femtoliter. Given that the cytosolic concentration of OG-1 was about $46 \mu \mathrm{M}$, and that injections of saturating amounts of calcium yield maximal fluorescence increases $\left(\Delta F / F_{\mathrm{o}}\right)$ of $4-5$, then a signal of $\Delta F / F_{\mathrm{o}}=1$ corresponds to binding of about $10 \mu \mathrm{M}$ of calcium to the indicator. The amount of calcium bound to indicator is approximately equal to that bound to endogenous buffers, and the amount of free calcium is small in comparison to that bound (Yao et al., 1995). Thus, 1 signal mass unit corresponds to $\sim 2 \times 10^{-20} \mathrm{~mol}$ of calcium (20 $\mu \mathrm{M}$ in a volume of 1 femtoliter); equivalent to a calcium current of about $4 \mathrm{pA}$ for $1 \mathrm{~ms}$.

Figure 5A shows averaged images of puffs recorded in control, and wild type and mutant PS1-expressing oocytes and illustrates the derivation of signal mass as a function of time during these events (Fig. 5B). Because calcium release during puffs is much faster than its subsequent removal from the cytosol (Sun et al., 1998) the signal mass reflects the accumulation of calcium in the cytosol over time, and its peak gives a measure of the total amount of calcium released. As is evident from Fig. 5B, the peak signal mass was potentiated in wtPS1-expressing cells relative to controls, and to an even greater extent in $\mathrm{PS}_{\mathrm{M}_{146 \mathrm{~V}}}$-expressing cells. Further analysis of 93-118 puffs per condition revealed that the mean amounts of calcium released during puffs in wtPS1- and PS1 $1_{\mathrm{M} 146 \mathrm{~V}}$-expressing cells were $153 \pm 13$ and $234 \pm 22 \%$ of that in controls, respectively (Fig. 5C).

\section{Increased Rates of Calcium Release Following PS1 Expression}

The release of greater amounts of calcium during puffs in mutant PS1-expressing cells could arise from a greater rate of calcium release, or because a given release rate was sustained for a longer time during each event. Since signal mass reflects the accumulation of calcium in the cytosol, the rate of rise of the signal mass (slopes of lines in Fig. 5B) can be used to estimate
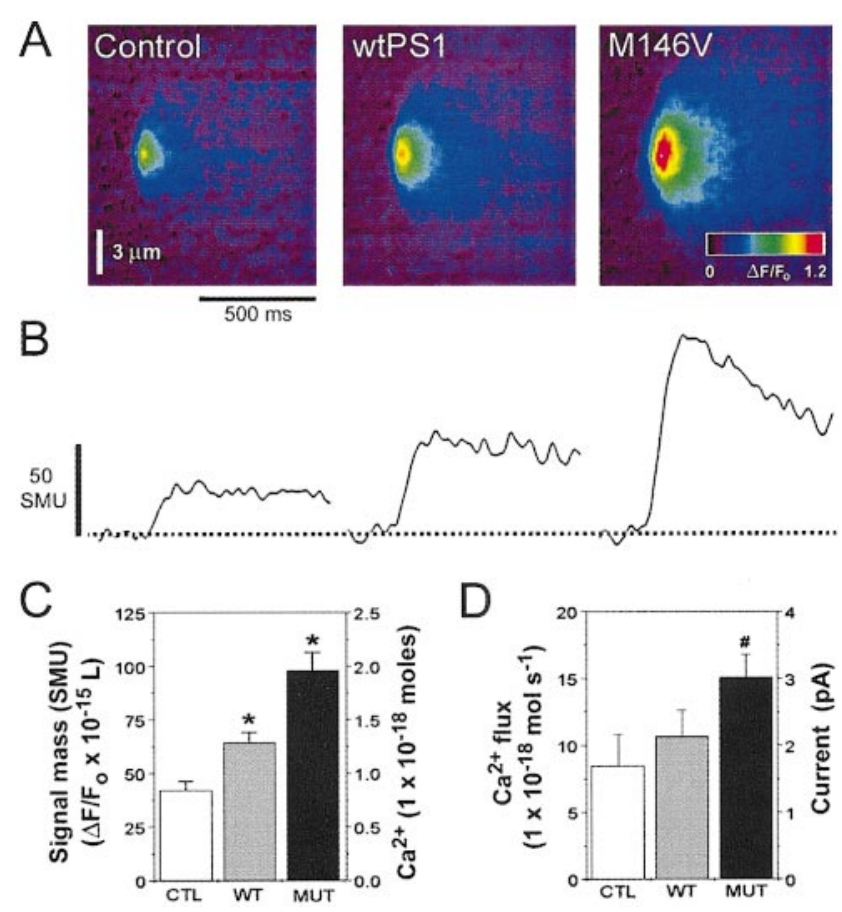

FIG. 5. PS1 potentiates calcium flux during puffs. (A) Averaged linescan images of calcium puffs from control cells and cells expressing wild type PS1 and $\mathrm{PS}_{\mathrm{M} 146 \mathrm{~V}}(12-14$ images per condition). (B) Signal mass time courses derived from the images in (A) using the procedure described in the text. (C) Mean peak signal mass associated with calcium puffs in control cells and cells expressing wild-type PS1 or PS1 $1_{\mathrm{M} 146 \mathrm{~V}}$ ( $n=93-118$ events per condition). Axes show calibrations both in units of signal mass (left) and moles of calcium (right). Signal mass values were significantly different $\left({ }^{*} P<\right.$ 0.01) between PS1-expressing cells and controls, and between wildtype PS1 and PS1 $1_{\mathrm{M} 146 \mathrm{~V}}$. (D) Corresponding estimates of calcium flux derived from the rate of rise of signal mass during elementary release events ( $n=25$ events per condition). Axes show calibrations in terms of moles of calcium per second (left) and calcium current (right). Calcium flux in $\mathrm{PS}_{\mathrm{M}_{146 \mathrm{~V}}}$-expressing cells was significantly different $(\# P<0.05)$ from controls.

the calcium release rate (i.e., calcium current through release channels; Sun et al., 1998)). Analysis of 25 events per condition showed that the rate of calcium release in wtPS1- and $\mathrm{PS1}_{\mathrm{M} 146 \mathrm{~V}}$-expressing cells was $126 \pm 24$ and $178 \pm 21 \%$, respectively, of that in controls (Fig. 5D). Based on the calculations detailed in the previous section, the calcium currents associated with puffs were approximately $1.7 \pm 0.5 \mathrm{pA}$ in control cells, and $2.1 \pm 0.4$ and $3.0 \pm 0.4 \mathrm{pA}$ in cells expressing wtPS1 or PS1 $1_{\mathrm{M} 146 \mathrm{~V}}$, respectively (Fig. 5D). Statistically, the rates of calcium release and corresponding currents were significantly different for $\mathrm{PS}_{\mathrm{M} 146 \mathrm{~V}}$-expressing cells relative to controls $(P<0.05)$, whereas calcium flux in wtPS1-expressing cells was slightly 
higher than controls, but not significantly so $(P>$ 0.05). Therefore, the potentiation of calcium puffs in $\mathrm{PS}_{\mathrm{M} 146 \mathrm{~V}}$ (and possibly wtPS1-)-expressing cells appears largely due to an increased rate of calcium release.

\section{Elevated ER Calcium Levels Following PS1 Expression}

One mechanism that could account for increased calcium flux is an elevation of calcium content within the ER. Such a scenario would be predicted to increase both the rate and the magnitude of calcium release associated with each puff, due to an increased driving force on calcium across the ER. To test this idea, oocytes expressing wtPS1 or PS1 $1_{\mathrm{M} 146 \mathrm{~V}}$ were treated with 1 $\mu \mathrm{M}$ thapsigargin in the nominal absence of calcium and stimulated with supramaximal $\mathrm{IP}_{3}$ concentrations at 90-s intervals; the cumulative amplitude of $\mathrm{IP}_{3^{-}}$ evoked fluorescence changes were then taken as a measure of ER calcium content (Fig. 6A). Alternative methods of determining ER calcium content, such as treatment with thapsigargin alone in calcium-free Ringer's, did not yield reliable estimates of the ER calcium content, possibly due to the rapid extrusion of cytosolic calcium from the cell (data not shown). As illustrated in Fig. 6B, the ER calcium content was found to be elevated in oocytes expressing wtPS1 and to a greater extent in cells expressing $\mathrm{PS}_{\mathrm{M} 146 \mathrm{~V}}$.

\section{PS1 Expression Does Not Affect the Density of ER Calcium Release Sites}

Although overall levels of $\mathrm{IP}_{3}$ receptor protein were similar in the different experimental conditions, it remained possible that their subcellular distribution was altered by presenilin expression. That is, presenilin expression might alter the number of functional $\mathrm{IP}_{3}$ receptor/channel complexes without altering the overall levels of protein. If this were the case, then the density of active calcium release channels would be expected to change as a consequence of presenilin expression. To address this possibility, we quantified the number of release sites present in the line-scan images from each condition, limiting our sample to those line-scan images taken between the threshold for puffs and the threshold for waves for each condition. The mean $( \pm \mathrm{SE})$ number of calcium release sites per $50-\mu \mathrm{m}$ linescan image was $6.1 \pm 0.9,5.9 \pm 0.7$, and $6.2 \pm 0.7$, for controls and wt PS1-expressing and $\mathrm{PS}_{\mathrm{M} 146 \mathrm{~V}}$-expressing cells, respectively. Therefore, no differences in the density of calcium release sites were
A
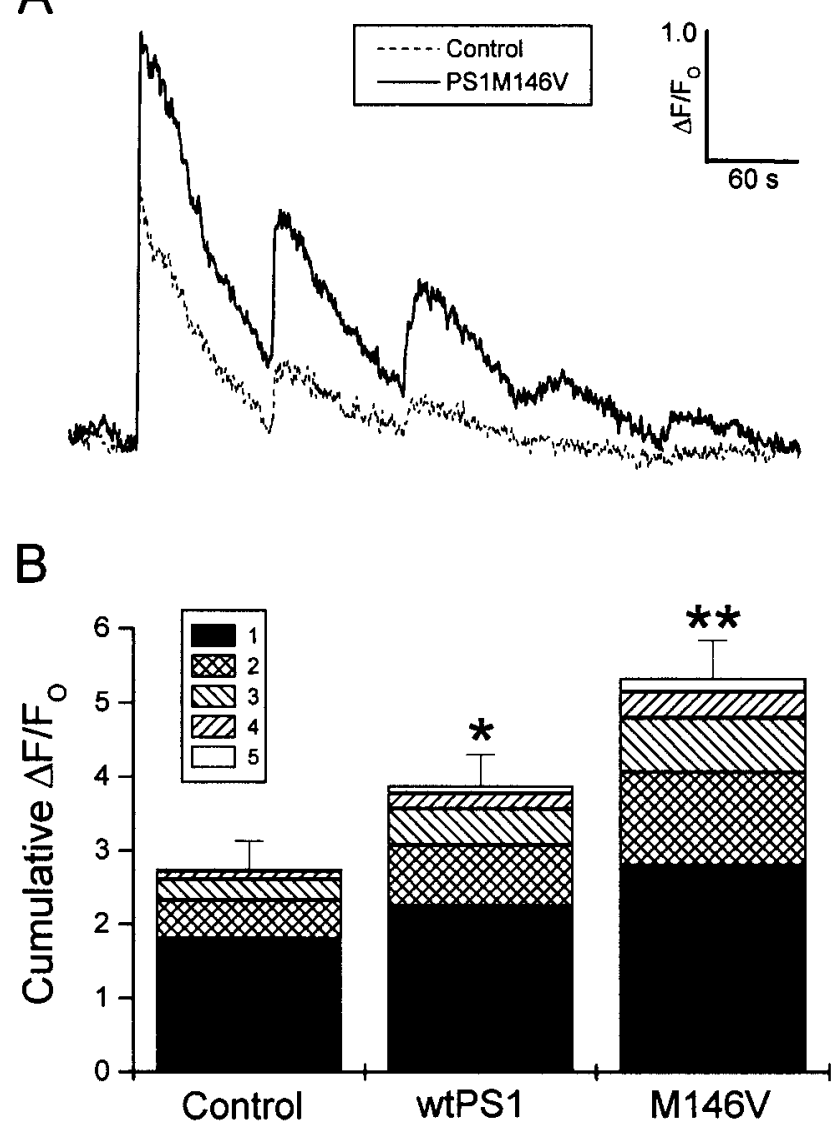

FIG. 6. PS1 increases ER calcium content. (A) Sample traces showing experimental protocol used to estimate ER calcium content. Cells loaded with caged $\mathrm{IP}_{3}$ and OG5-N were treated with $1 \mu \mathrm{M}$ thapsigargin in calcium-free Ringer's solution and stimulated with supramaximal (200 ms) UV flashes at 90-s intervals. Note that sample recordings from wtPS1-expressing cells have been omitted for clarity. (B) Cumulative changes in fluorescence amplitude ( $\Delta F$ / $F_{\mathrm{o}}$ ) taken from 10-11 cells per condition. The numbers (1-5) in the figure legend refer to successive rounds of UV flash photolysis. ${ }^{*} \mathrm{P}<$ 0.05 relative to $\mathrm{H}_{2} \mathrm{O}$-injected controls; ${ }^{* *} \mathrm{P}<0.01$ relative to controls and relative to wtPS1-expressing cells.

detected. Taken together with the other findings of this study, these data suggest that presenilin expression affects the activity, but not the amount, of $\mathrm{IP}_{3}$ receptor/channel complexes. The altered activity of these channels, in turn, is largely attributable to alterations in the content of ER calcium stores.

\section{DISCUSSION}

Although numerous studies have shown that global calcium signals are enhanced in cells expressing pre- 
senilin mutations, the subcellular mechanisms underlying these potentially pathogenic changes are poorly understood. To address this important issue, we studied calcium release mechanisms using high-resolution confocal microscopy in Xenopus oocytes expressing wild type or mutant PS1. The present study goes beyond previous studies in two important respects. First, by examining the fundamental units of calcium release, this study has resolved in unprecedented detail the fine-scale kinetics of the alterations in ER calcium signaling conferred by presenilin mutations. Second, by examining a variety of potential subcellular mechanisms responsible for these changes in a single, model system, this study has identified the subcellular basis for presenilin-mediated modulation of calcium signaling. Here we demonstrate that the effects of presenilin mutations on calcium signaling are largely attributable to changes in the fundamental units of calcium release (puffs) making up global calcium signals. Notably, we did not observe any changes in the expression levels of calcium release channels. Thus, in this system, the potentiation of global calcium signals conferred by mutant PS1 is caused by an increase in the magnitude of calcium puffs themselves rather than in the number of $\mathrm{IP}_{3}$-mediated release channels.

The mechanism producing calcium puffs in cells expressing wild-type or mutant PS1 also exhibited an enhanced sensitivity to $\mathrm{IP}_{3}$. A similar effect has been observed in other experimental systems, albeit at the level of global calcium signals. For instance, FAD fibroblasts harboring PS1 mutations are more sensitive to agonists coupled to $\mathrm{IP}_{3}$ (Hirashima et al., 1996). Although changes in numerous components of the complex phosphoinositide signal transduction cascade might account for these alterations (e.g., levels or activity of G-proteins, phospholipase C, etc.), a similar enhancement of sensitivity has been observed in $\mathrm{Xe}$ nopus oocytes expressing mutations in PS1 and PS2 following direct photorelease of $\mathrm{IP}_{3}$ from a caged precursor (Leissring et al., 1999a, 1999b). These findings, together with the present results, suggest that the enhanced sensitivity to $\mathrm{IP}_{3}$ is manifested at the level of calcium release from the ER and is not (exclusively) due to changes in upstream elements of the phosphoinositide second-messenger pathway.

The potentiation of puffs is largely attributable to an increase in the rate of calcium release during each event, which in turn appears to be due to elevated levels of calcium in the lumen of the ER. Additional evidence supports a role for the presenilins in regulating ER calcium levels. First, thapsigargin- and ionophore-releasable calcium pools are increased in sev- eral systems harboring PS1 mutations, including PC12 cells (Guo et al., 1996), fibroblasts from human FAD patients (Gibson et al., 1996) and mutant PS1 knock-in animals (Leissring et al., 2000a). Second, in brain slices from transgenic animals expressing PS1 mutations, electrophysiological abnormalities are present, including enhanced long-term potentiation (LTP) and increased after-hyperpolarization, and both alterations can be attributed to increased release of ER calcium (Barrow et al., 2000; Parent et al., 1999). Finally, PS1linked FAD fibroblasts express elevated levels of acylphosphatase (Liguri et al., 1996), an enzyme that modulates the activity of the ER calcium ATPases responsible for transporting calcium into the lumen of the ER, suggesting that altered activity of these enzymes could explain the elevation of ER calcium levels.

In previous work, our laboratory demonstrated that fibroblasts from mutant PS1 knock-in mice show deficits in CCE (Leissring et al., 2000a) and we have observed similar disturbances in Xenopus oocytes expressing presenilin mutations (Leissring, Parker, and LaFerla, unpublished observations; see also Fig. 4 in Leissring et al., 1999b). To trigger CCE, calcium levels in the ER must drop below a certain threshold (Fierro $\&$ Parekh, 2000). Our findings suggest that the triggering of CCE is impaired in cells harboring presenilin mutations because abnormally high ER calcium levels prevent calcium levels from dropping below the threshold required to activate CCE (see Leissring et al., 2000a). The finding that mutant presenilin causes an over-elevation of ER calcium stores therefore provides a succinct mechanism to account for the two principal calcium signaling alterations conferred by presenilin mutations: the potentiation of ER calcium release and deficits in CCE.

$\mathrm{IP}_{3}$-mediated cellular calcium signaling operates in two different spatio-temporal modes: (i) local signals carried by the high, transient calcium levels generated by elementary release events that selectively activate closely adjacent effectors; and (ii) longer range global signals that result from the summation of calcium released at numerous puff sites to produce a lower average calcium elevation throughout the cell. These modes permit specificity in calcium signaling. In particular, the affinity of the calcium uptake system in mitochondria, which exist in close apposition to ER calcium release sites (Rizzuto et al., 1993), is too low for these organelles to detect global elevations in calcium concentration (usually $<1 \mu \mathrm{M}$ ), whereas they respond avidly to the much larger calcium signals near elementary events (Berridge, 1998; Hajnoczky et 
al., 1995; Rizzuto et al., 1993). Overloading of mitochondrial calcium is implicated in apoptosis (Berridge et al., 1998; Mattson et al., 2000a; Nicholls \& Budd, 2000) and PS1 mutations also lead to mitochondrial dysfunction, an effect that can be attenuated by agents that inhibit release of calcium from the ER (Keller et al., 1998; Mattson et al., 2000b). Our finding that mutant PS1 expression results in much greater calcium liberation during elementary puffs therefore raises the possibility that the disruptions in local calcium signaling processes that we have described could contribute to the enhanced vulnerability to cell death conferred by presenilin mutations. An alternative possibility not addressed in the present study is that impaired mitochondrial calcium buffering could contribute to the increased puff size conferred by presenilins.

A particularly interesting question for future research involves the relationship between $\gamma$-secretase mediated cleavage of APP and altered calcium signaling, particularly since alterations in both of these processes appear to be invariant consequences of presenilin mutations. One possibility is that $\beta$-amyloid is a necessary mediator of the calcium alterations. To address this question in the future, we have crossed mutant PS1 knock-in animals with APP knock-out mice; the presence or absence of calcium signaling alterations in these animals will confute or confirm, respectively, this idea. Another possibility is that the observed calcium signaling alterations can affect $\gamma$-secretase activity. In support of this idea, a recent report showed that an inhibitor of CCE caused increased production of the more amyloidogenic form of $\beta$-amyloid (Yoo et al., 2000). Finally, it is formally possible that calcium signaling alterations and increased $\beta$-amyloid production are independent consequences of presenilin mutations. Again, the cross of mutant PS1 knock-in animals with APP knock-out animals will be useful in resolving this question.

In sum, the present study has examined a number of possible mechanisms to explain the effects of presenilin mutations on calcium signaling. We conclude that mutant presenilins' effects on calcium signals are predominantly attributable to an abnormal overfilling intracellular calcium pools. Given the likely pathogenic consequences of perturbed calcium homeostasis, therapies which counteract these changes could possess significant therapeutic benefit. Intriguingly, the potentiation of calcium signaling by PS1 can be reversed by co-expression with calsenilin, a calcium-binding protein that associates with the C-terminus of the presenilins (Leissring et al., 2000b). Thus, future research into the possible effects of calsenilin (and other prese- nilin-associated proteins) on ER calcium homeostasis could help to resolve the molecular mechanisms by which mutant presenilins perturb calcium signaling pathways and potentially other AD-related pathogenic events such as APP proteolysis and hyperphosphorylation of tau.

\section{ACKNOWLEDGMENTS}

This work was supported by grants from the National Institutes of Health (GM 48071, AG15409, AG16573), NRSA AG00096-17, the American Health Assistance Foundation, and the American Federation for Aging Research. We thank Tritia Yamasaki for expert technical assistance and Yama Akbari for critical reading of the manuscript.

\section{REFERENCES}

Barrow, P. A., Empson, R. M., Gladwell, S. J., Anderson, C. M., Killick, R., Yu, X., Jefferys, J. G., \& Duff, K. (2000) Functional phenotype in transgenic mice expressing mutant human presenilin-1. Neurobiol. Dis. 7, 119-126.

Berridge, M. J. (1995) Capacitative calcium entry. Biochem. J. 312, $1-11$.

Berridge, M. J. (1997) Elementary and global aspects of calcium signalling. J. Exp. Biol. 200, 315-319.

Berridge, M. J. (1998) Neuronal calcium signaling. Neuron 21, 13-26.

Berridge, M. J., Bootman, M. D., \& Lipp, P. (1998) Calcium-A life and death signal. Nature 395, 645-648.

Callamaras, N., \& Parker, I. (1998) Caged inositol 1,4,5-trisphosphate for studying release of $\mathrm{Ca}^{2+}$ from intracellular stores. Methods Enzymol. 291, 380-403.

Callamaras, N., \& Parker, I. (1999) Construction of line-scan confocal microscope for physiological recording. Methods Enzymol. 307, 152-169.

Clark, R. F., Hutton, M., Fuldne, R. A. S. F., Karran, E., Talbot, C., Crook, R., Lendon, C., Prihar, G., He, C., et al. (1995) The structure of the presenilin 1 (S182) gene and identification of six novel mutations in early onset AD families. Alzheimer's disease collaborative group. Nature Genet. 11, 219-222.

Etcheberrigaray, R., Hirashima, N., Nee, L., Prince, J., Govoni, S., Racchi, M., Tanzi, R. E., \& Alkon, D. L. (1998) Calcium responses in fibroblasts from asymptomatic members of Alzheimer's disease families. Neurobiol. Dis. 5, 37-45.

Gibson, G. E., Zhang, H., Toral-Barza, L., Szolosi, S., \& Tofel-Grehl, B. (1996) Calcium stores in cultured fibroblasts and their changes with Alzheimer's disease. Biochim. Biophys. Acta 1316, 71-77.

Guo, Q., Furukawa, K., Sopher, B. L., Pham, D. G., Xie, J., Robinson, N., Martin, G. M., \& Mattson, M. P. (1996) Alzheimer's PS-1 mutation perturbs calcium homeostasis and sensitizes PC12 cells to death induced by amyloid beta-peptide. Neuroreport 8, 379-383.

Hajnoczky, G., Robb-Gaspers, L. D., Seitz, M. B., \& Thomas, A. P. (1995) Decoding of cytosolic calcium oscillations in the mitochondria. Cell 82, 415-424.

Hirashima, N., Etcheberrigaray, R., Bergamaschi, S., Racchi, M., Battaini, F., Binetti, G., Govoni, S., \& Alkon, D. L. (1996) Calcium 
responses in human fibroblasts: A diagnostic molecular profile for Alzheimer's disease. Neurobiol. Aging 17, 549-555.

Ito, E., Oka, K., Etcheberrigaray, R., Nelson, T. J., McPhie, D. L., Tofel-Grehl, B., Gibson, G. E., \& Alkon, D. L. (1994) Internal Ca ${ }^{2+}$ mobilization is altered in fibroblasts from patients with Alzheimer disease. Proc. Natl. Acad. Sci. USA 91, 534-538.

Keller, J. N., Guo, Q., Holtsberg, F. W., Bruce-Keller, A. J., \& Mattson, M. P. (1998) Increased sensitivity to mitochondrial toxininduced apoptosis in neural cells expressing mutant presenilin-1 is linked to perturbed calcium homeostasis and enhanced oxyradical production. J. Neurosci. 18, 4439-4450.

Kovacs, D. M., Fausett, H. J., Page, K. J., Kim, T. W., Moir, R. D., Merriam, D. E., Hollister, R. D., Hallmark, O. G., Mancini, R., Felsenstein, K. M., et al. (1996) Alzheimer-associated presenilins 1 and 2: Neuronal expression in brain and localization to intracellular membranes in mammalian cells. Nature Med. 2, 224-229.

Kume, S., Muto, A., Aruga, J., Nakagawa, T., Michikawa, T., Furuichi, T., Nakade, S., Okano, H., \& Mikoshiba, K. (1993) The Xenopus $\mathrm{IP}_{3}$ receptor: Structure, function, and localization in oocytes and eggs. Cell 73, 555-570.

Leissring, M. A., Akbari, Y., Fanger, C. M., Cahalan, M. D., Mattson, M. P., \& LaFerla, F. M. (2000a) Capacitative calcium entry deficits and elevated luminal calcium content in mutant presenilin-1 knockin mice. J. Cell Biology 149, 793-798.

Leissring, M. A., Parker, I., \& LaFerla, F. M. (1999a) Presenilin-2 mutations modulate amplitude and kinetics of inositol 1,4,5trisphosphate-mediated calcium signals. J. Biol. Chem. 274, 3253532538.

Leissring, M. A., Paul, B. A., Parker, I., Cotman, C. W., \& LaFerla, F. M. (1999b) Alzheimer's presenilin-1 mutation potentiates inositol 1,4,5-trisphosphate-mediated calcium signaling in Xenopus oocytes. J. Neurochem. 72, 1061-1068.

Leissring, M. A., Yamasaki, T. R., Wasco, W., Buxbaum, J. D., Parker, I., \& LaFerla, F. M. (2000b) Calsenilin reverses presenilinmediated enhancement of calcium signaling. Proc. Natl. Acad. Sci. USA 97, 8590-8593.

Liguri, G., Cecchi, C., Latorraca, S., Pieri, A., Sorbi, S., Degl'Innocenti, D., \& Ramponi, G. (1996) Alteration of acylphosphatase levels in familial Alzheimer's disease fibroblasts with presenilin gene mutations. Neurosci. Lett. 210, 153-156.

Mattson, M. P., LaFerla, F. M., Chan, S. L., Leissring, M. A., Shepel, P. N., \& Geiger, J. D. (2000a) Calcium signaling in the ER: Its role in neuronal plasticity and neurodegenerative disorders. Trends Neurosci. 23, 222-229.
Mattson, M. P., Zhu, H., Yu, J., \& Kindy, M. S. (2000b) Presenilin-1 mutation increases neuronal vulnerability to focal ischemia in vivo and to hypoxia and glucose deprivation in cell culture: Involvement of perturbed calcium homeostasis. J. Neurosci. 20, 13581364.

Nicholls, D. G., \& Budd, S. L. (2000) Mitochondria and neuronal survival. Physiol. Rev. 80, 315-360.

Parent, A., Linden, D. J., Sisodia, S. S., \& Borchelt, D. R. (1999) Synaptic transmission and hippocampal long-term potentiation in transgenic mice expressing FAD-linked presenilin 1. Neurobiol. Dis. 6, 56-62.

Parker, I., Choi, J., \& Yao, Y. (1996) Elementary events of Insp3induced $\mathrm{Ca}^{2+}$ liberation in Xenopus oocytes: Hot spots, puffs and blips. Cell Calcium 20, 105-121.

Rizzuto, R., Brini, M., Murgia, M., \& Pozzan, T. (1993) Microdomains with high $\mathrm{Ca}^{2+}$ close to $\mathrm{IP}_{3}$-sensitive channels that are sensed by neighboring mitochondria. Science 262, 744-747.

Selkoe, D. J. (1999) Translating cell biology into therapeutic advances in Alzheimer's disease. Nature 399, A23-31.

Sun, X. P., Callamaras, N., Marchant, J. S., \& Parker, I. (1998) A continuum of InsP3-mediated elementary $\mathrm{Ca}^{2+}$ signalling events in Xenopus oocytes. J. Physiol. (London) 509, 67-80.

Thinakaran, G., Borchelt, D. R., Lee, M. K., Slunt, H. H., Spitzer, L., Kim, G., Ratovitsky, T., Davenport, F., Nordstedt, C., Seeger, M., et al. (1996) Endoproteolysis of presenilin 1 and accumulation of processed derivatives in vivo. Neuron 17, 181-190.

Thinakaran, G., Regard, J. B., Bouton, C. M., Harris, C. L., Price, D. L., Borchelt, D. R., \& Sisodia, S. S. (1998) Stable association of presenilin derivatives and absence of presenilin interactions with APP. Neurobiol. Dis. 4, 438-453.

Weber, L. L., Leissring, M. A., Yang, A. J., Glabe, C. G., Cribbs, D. H., \& LaFerla, F. M. (1997) Presenilin-1 immunoreactivity is localized intracellularly in Alzheimer's disease brain, but not detected in amyloid plaques. Exp. Neurol. 143, 37-44.

Yao, Y., Choi, J., \& Parker, I. (1995) Quantal puffs of intracellular $\mathrm{Ca}^{2+}$ evoked by inositol trisphosphate in Xenopus oocytes. J. Physiol. (London) 482, 533-553.

Yoo, A. S., Cheng, I., Chung, S., Grenfell, T. Z., Lee, H., Pack-Chung, E., Handler, M., Shen, J., Xia, W., Tesco, G., et al. (2000) Presenilinmediated modulation of capacitative calcium entry. Neuron 27, 561-572.

Zhang, J., Kang, D. E., Xia, W., Okochi, M., Mori, H., Selkoe, D. J., \& Koo, E. H. (1998) Subcellular distribution and turnover of presenilins in transfected cells. J. Biol. Chem. 273, 12436-12442. 